\title{
A!
}

This is an electronic reprint of the original article.

This reprint may differ from the original in pagination and typographic detail.

Wang, Xiyu; Ali, Yusein; Jäntti, Riku

\section{A Simplified Multi-Antenna Receiver for General Binary-Modulated Ambient Backscatter Signal}

Published in:

2021 IEEE Global Communications Conference (GLOBECOM)

DOI:

10.1109/GLOBECOM46510.2021.9685353

Published: 01/02/2022

Document Version

Peer reviewed version

Please cite the original version:

Wang, X., Ali, Y., \& Jäntti, R. (2022). A Simplified Multi-Antenna Receiver for General Binary-Modulated Ambient Backscatter Signal. In 2021 IEEE Global Communications Conference (GLOBECOM) (pp. 1-6). IEEE. https://doi.org/10.1109/GLOBECOM46510.2021.9685353

This material is protected by copyright and other intellectual property rights, and duplication or sale of all or part of any of the repository collections is not permitted, except that material may be duplicated by you for your research use or educational purposes in electronic or print form. You must obtain permission for any other use. Electronic or print copies may not be offered, whether for sale or otherwise to anyone who is not an authorised user. 


\title{
A Simplified Multi-Antenna Receiver for General Binary-Modulated Ambient Backscatter Signal
}

\author{
Xiyu Wang, Hüseyin Yiğitler, and Riku Jäntti \\ Department of Communications and Networking, Aalto University, 02150 Espoo, Finland \\ E-mail: \{firstname.surname $\}$ aalto.fi
}

\begin{abstract}
Multi-antenna receivers are becoming popular in ambient backscatter communication (AmBC) because they help to improve the detection performance by improving the signalto-interference-plus-noise ratio (SINR) of the backscatter signal. Recent solutions, however, suffer from high implementation complexity and they tend to require the receiver to have high dynamic range. In this paper, a simplified receiver is proposed, which is able to overcome the above limitations. The receivers for the deterministic-unknown ambient signal and the Gaussian signal are derived using maximum-a-posteriori (MAP) criterion. Then, their detection performances are derived for bit-error-rate analysis. It is shown that the test statistic of the receiver is independent of the ambient signal type. The components for implementation are halved compared with the optimum AmBC receiver at the cost of an SINR penalty as low as 1-dB to achieve the same detection performance for the deterministic ambient signal and 3.6-dB for the Gaussian ambient signal. The dynamic range problem can be mitigated by implementing it fully in the analog domain. By providing attractive practical advantages mentioned above, the proposed simplified receiver, therefore, enables cost-effective $\mathrm{AmBC}$ receiver implementations.

Index Terms-Ambient backscatter communication, Internetof-things, receiver design, performance analysis
\end{abstract}

\section{INTRODUCTION}

Ambient backscatter communication $(\mathrm{AmBC})$ is envisioned to be an important component for realizing low-power ubiquitous connections in the Internet-of-things (IoT) deployment. Since the AmBC technology requires neither the power-hungry amplifiers nor a dedicated carrier illuminator which generates dedicated carrier signals for devices [1], [2], it overcomes the limitations of spectrum resources and energy resources resulting from a large number of connections.

In an AmBC system, the passive backscatter device (BD) transmits its information by modulating the signal on top of the ambient signal existing in the air and scattering the modulated signal to the receiver. The receiver detects the BD signal among a composite of two paths of signal, namely the direct path which is not affected by the BD, and the backscatter path that carries BD signal. However, the detection performance is hampered by the inherent low signal-to-interference-plus-noise ratio (SINR) of the backscatter signal. The reason is that the dyadic backscatter path suffers from a tremendous direct path interference (DPI) and an additional fading that is induced by the unknown and fast-varying ambient signal and the unknown channels. Therefore, it is necessary to address these issues to obtain an acceptable detection performance.
The strong DPI is mitigated by either differentiating direction [3], [4] or spectrum [5] of two paths of signal or using complex signal processing methods [6]. Among the approaches, separating the directions of signal, which is realized by multiantenna receivers, achieves a higher degree of freedom of implementation [7] and acquires an array gain to the received signal. Hence, in this paper, we consider the multi-antenna AmBC receivers.

When utilizing the on-off keying (OOK) modulation at $\mathrm{BD}$, the seminal work [2] detects the $\mathrm{BD}$ signal from the ratio between the amplitudes of the received signal at two antennas. The works [5] and [8] investigate the energy detector by leveraging the special structure of orthogonal frequency division multiplexing (OFDM) signal. In order to achieve a signal-to-noise ratio (SNR) gain for the demodulation, the binary phase shift keying (BPSK) modulation is considered at the BD. The work [7] studies the multi-antenna receiver for the BPSK-modulated backscatter signal with Gaussian ambient signal. The recent work [4] derives the optimum receiver for any binary-modulated BD signal, which is a generalization of the existing receivers in [7], and [3], [9] which consider the OOK-modulated BD signal and Gaussian ambient signal. The work [4] extends the receiver design when no prior information about ambient signal is available. The above receivers are noncoherent in nature. If the BD signal is demodulated coherently, however, a more complicated receiver is required [10].

Available AmBC multi-antenna receivers have practical issues for implementation. For instance, they require the analogto-digital converter (ADC) to have a high dynamic range if they are implemented in the digital domain. This is because the direct path signal is much stronger than the backscatter path signal. The ADC, thus, needs a high dynamic range to capture more information that is buried in the few least significant bits [4], [7]. Although a hybrid method is provided in [4] to overcome the dynamic range problem, the optimum receiver has to implement the same processing stages two times.

For overcoming the practical issues, in this paper, we propose a cost-effective multi-antenna simplified receiver for two types of ambient signal: i) deterministic-unknown ambient signal and ii) Gaussian ambient signal. The receiver is derived from an intermediate measurement of the optimum receiver using maximum-a-posteriori (MAP) criterion. The test statistic is independent of the ambient signal type. The receiver only needs one beamformer, which optimizes the difference of signal energy under two hypotheses inside the subspace orthogonal 
to one signal direction. The simplified receiver halves the components for implementation at the cost of a small bit-errorrate (BER) performance penalty and, can mitigate the dynamic range problem by implementing it in the analog domain. Our contributions in this paper can be summarized as follows:

- We propose the multi-antenna AmBC simplified receiver that demodulates any binary-modulated backscatter signal for the deterministic-unknown ambient signal and the Gaussian ambient signal. The derived receiver is non-coherent. We show that the test statistic does not rely on the type of the ambient signal, but the decision threshold varies.

- We derive the error probability of the simplified receiver for two types of ambient signal. We show that the simplified receiver achieves the same performance as the optimum receiver with 1-dB and 3.6-dB more SNR for the deterministicunknown and the Gaussian ambient signal, respectively.

- We present the practical implementation of the simplified receiver and show its advantages. We provide one estimation method for the beamformer and the threshold, and discuss the key parameter affecting the receiver performance. The receiver performance in terms of BER shows that the derived theoretical error probabilities are valid, the estimation method is effective, and the simplified receiver only has a small performance loss compared with the optimum receiver.

The remainder of the paper is organized as follows. In Section II, a general system model is given and the optimum receiver is reviewed. The simplified receiver and its performance metric are derived in Section III. The practical implementation issues are provided in Section IV. Simulation results are presented and discussed in Section V. Finally, conclusions are drawn in Section VI.

\section{BACKGROUND}

\section{A. System model}

We consider a typical bi-static AmBC deployment in which a single-antenna legacy system transmitter (Tx) transmits the ambient signal to serve its own users, a single-antenna backscatter device (BD) modulates its own information on top of the incident ambient signal and scatters the modulated signal that is received by the AmBC receiver (Rx) with a $N_{r^{-}}$ antenna array. We select the $\left\lceil N_{r} / 2\right\rceil$ th element of the $\mathrm{Rx}$ array as the reference antenna, where $\lceil\cdot\rceil$ is the ceiling function. As shown in Fig. 1, three nodes are in a two-dimensional Cartesian coordinate system, where the $x$-axis is the line through the $\mathrm{Tx}$ and the $\mathrm{Rx}$ reference antenna, and the origin point is the midpoint of line segment between the Tx and the Rx reference antenna. The coordinates of the Tx antenna and the $l$ th Rx antenna and the BD antenna are $\boldsymbol{p}_{t}, \boldsymbol{p}_{r l}$ and $\boldsymbol{p}$, respectively. The distances between three nodes are represented as $d_{0 l}=\left\|\boldsymbol{p}_{t}-\boldsymbol{p}_{r l}\right\|, \quad d_{1 l}=\left\|\boldsymbol{p}-\boldsymbol{p}_{r l}\right\|$, and $\quad d_{2}=\left\|\boldsymbol{p}_{t}-\boldsymbol{p}\right\|$.

Let $\boldsymbol{\alpha}=\left[\alpha_{1}, \cdots, \alpha_{N_{r}}\right]$ and $\boldsymbol{\beta}=\left[\beta_{1}, \cdots, \beta_{N_{r}}\right]$ denote the channel gain vectors of the direct path and of the backscatter path, where $\alpha_{l}$ and $\beta_{l}$ are the channel gains of two paths seen by the $l$ th antenna. The channel $\boldsymbol{\alpha}$ is the sum of multipath components that are not affected by the BD operation,

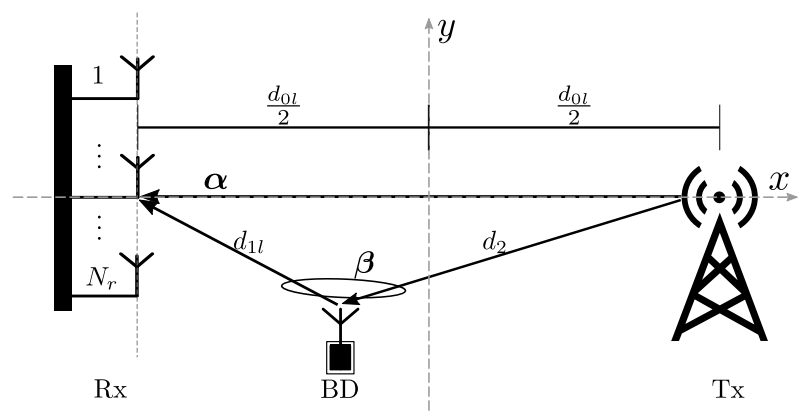

Fig. 1. An illustration of the AmBC system.

and channel $\boldsymbol{\beta}$ contains all the multi-path components that are affected by the BD operation ${ }^{1}$. We assume the quasistatic scenario where the channels stay constant during the transmission of one block [11, Sec. 5.4.1], but may vary from one block to another. The average path-losses of direct path and backscatter path based on deployment and geometry in the presence of line-of-sight (LOS) are given as

$$
E\left\{\left|\alpha_{l}\right|^{2}\right\}=\left(\frac{c_{0} / f_{c}}{4 \pi d_{0 l}}\right)^{2}, E\left\{\left|\beta_{l}\right|^{2}\right\}=\rho\left(\frac{c_{0} / f_{c}}{4 \pi d_{1 l}}\right)^{2}\left(\frac{c_{0} / f_{c}}{4 \pi d_{2}}\right)^{2} .
$$

where $f_{c}$ is the carrier frequency, $c_{0}$ denotes the free-space electromagnetic propagation speed and $0<\rho<1$ is the power loss induced by the implementation of BD modulator ${ }^{2}$.

Let $s$ be the unknown ambient signal transmitted from the Tx and $x$ be the signal transmitted by the BD. The $k$ th sample of received signal at the Rx antenna array can be written as

$$
\boldsymbol{y}[k]=\boldsymbol{\alpha} s[k]+\boldsymbol{\beta} s[k] x[k]+\boldsymbol{n}[k],
$$

where $\boldsymbol{n}[k] \sim \mathcal{C N}(\mathbf{0}, \boldsymbol{I})$ is the complex Gaussian noise, whose components are independent of $s$ and $x$. In this work, we consider the binary-modulated BD signal, i.e., $x \in\left\{x_{0}, x_{1}\right\}$. The ambient signal $s$ is unknown to be the $\mathrm{Rx}$ in practice due to the little cooperation between the legacy system and the AmBC system. Therefore, we consider two cases of $s$ : i) when there is no prior information about $s, s$ is treated as an unknown constant, which is referred to as deterministic-unknown s; and ii) the prior information is available at the $\mathrm{Rx}, s$ is assumed to be a zero-mean Gaussian variable with constant variance, i.e., $s \sim \mathcal{C N}\left(0, \sigma_{s}^{2}\right)$, which is close approximation for some real-world physical signals with complex modulation.

The averaged received SNR of the direct path signal at the lth antenna is $E\left\{\left|\alpha_{l}\right|^{2}\right\} E\left\{|s|^{2}\right\}$ since the noise power is normalized. We denote the SNR of the direct path signal averaged over $N_{r}$ antenna components is $\gamma=E\left\{\|\boldsymbol{\alpha}\|^{2}\right\} E\left\{|s|^{2}\right\} / N_{r}$. Following this definition, the transmit power of ambient signal can be given as $P_{s}=\gamma N_{r} / E\left\{\|\boldsymbol{\alpha}\|^{2}\right\}$. Specifically, for the deterministic-unknown $s, P_{s}=|s|^{2}$, and for the Gaussian $s$, $P_{s}=\sigma_{s}^{2}$. Then, the effective SNR of the backscatter signal is given by $\gamma_{e}=\gamma E\left\{\|\boldsymbol{\beta}\|^{2}\right\} / E\left\{\|\boldsymbol{\alpha}\|^{2}\right\}$.

\footnotetext{
${ }^{1}$ The structural mode of the BD antenna is included in the channel $\boldsymbol{\alpha}$ since it is independent of BD operation.

${ }^{2}$ We assume the BD harvested enough energy for modulating its signal. The power loss mainly arises from the polarization mismatch of the $\mathrm{BD}$ antenna.
} 
We assume the duration of $x$ is longer than that of $s$ so that each temporal sample of $\boldsymbol{y}$ is affected by independent symbol of $s$. We also assume the Rx and the BD are perfectly synchronized. In what follows, we drop the time dependence of $\boldsymbol{y}$ since the analysis of the receiver is based on one temporal sample of the received signal. The signal sample received at the $\mathrm{Rx}$ can be rewritten as

$$
\boldsymbol{y}=\left(\boldsymbol{\alpha}+x_{i} \boldsymbol{\beta}\right) s+\boldsymbol{n}=\boldsymbol{g}_{i} s+\boldsymbol{n},
$$

where we denote $\boldsymbol{g}_{i}=\boldsymbol{\alpha}+x_{i} \boldsymbol{\beta}$ as the signal direction.

\section{B. Preliminary}

The optimum multi-antenna receiver for general binarymodulated backscatter signal proposed in [4] is derived from the maximum-a-posteriori (MAP) criterion for both the deterministic-unknown $s$ and the Gaussian $s$. The equivalence of the optimum receivers for two types of ambient signal under high SNR condition has been shown. The test statistic of the optimum receiver contains two beamforming matrices $\boldsymbol{G}_{i}, i \in\{0,1\}$, which is expressed as

$$
z_{o p t}=\boldsymbol{y}^{H}\left(\boldsymbol{G}_{\mathbf{0}}-\boldsymbol{G}_{\mathbf{1}}\right) \boldsymbol{y},
$$

where the beamforming matrix

$$
\boldsymbol{G}_{i}=\boldsymbol{I}-\frac{\boldsymbol{g}_{i} \boldsymbol{g}_{i}^{H}}{\left\|\boldsymbol{g}_{i}\right\|^{2}}
$$

where $\boldsymbol{I}$ is the identity matrix so that $\boldsymbol{G}_{i}$ is the orthogonal projection matrix of $\boldsymbol{g}_{i}$. In order to simplify it, we dispose of one beamforming matrix, say $\boldsymbol{G}_{1}$, and design the receiver with respect to the other subspace, $\boldsymbol{G}_{0}$.

\section{SIMPLIFIED RECEIVER}

In this section, the simplified receiver structures are derived for the deterministic-unknown ambient signal and the Gaussian ambient signal, and their performances are analyzed.

\section{A. Receiver structure}

We derive the simplified receiver based on the measurement that falls in the subspace projected by the beamforming matrix $\boldsymbol{G}_{0}$, that is

$$
\tilde{\boldsymbol{y}}=\boldsymbol{G}_{0} \boldsymbol{y}=\boldsymbol{G}_{0} \boldsymbol{g}_{i} s+\boldsymbol{G}_{0} \boldsymbol{n} .
$$

Then, the receiver makes a decision of the $\mathrm{BD}$ signal given the measurement by using the MAP criterion. By applying the Bayes' theorem, the MAP criterion can be written as

$$
f\left(x_{0} \mid \tilde{\boldsymbol{y}}\right) \underset{\mathcal{H}_{1}}{\stackrel{\mathcal{H}_{0}}{\gtrless}} f\left(x_{1} \mid \tilde{\boldsymbol{y}}\right) \Longrightarrow f\left(\tilde{\boldsymbol{y}} \mid x_{0}\right) \underset{\mathcal{H}_{1}}{\stackrel{\mathcal{H}_{0}}{\gtrless}} \frac{p\left(x_{1}\right)}{p\left(x_{0}\right)} f\left(\tilde{\boldsymbol{y}} \mid x_{1}\right)
$$

where $\mathcal{H}_{0}$ and $\mathcal{H}_{1}$ denote the null-hypothesis and its alternative, respectively, $f\left(x_{i} \mid \tilde{\boldsymbol{y}}\right)$ is the posterior probability of the BD signal $x_{i}$ given $\tilde{\boldsymbol{y}}, p\left(x_{i}\right)$ is the prior probability of $x_{i}$ and $f\left(\tilde{\boldsymbol{y}} \mid x_{i}\right)$ is the likelihood function of $\tilde{\boldsymbol{y}}$ when $x_{i}$ is transmitted.

For the AmBC system, the ambient signal $s$ is unknown to the receiver as the legacy receiver is implemented independently with the $\mathrm{AmBC}$ receiver. In this case, the measurement $\tilde{\boldsymbol{y}}$ given $x_{i}$ is transmitted has a joint distribution of the ambient signal and the noise. Hence, $f\left(\tilde{\boldsymbol{y}} \mid x_{i}\right)$ is given by

$$
f\left(\tilde{\boldsymbol{y}} \mid x_{i}\right)=\int_{\mathbb{S}} f\left(\tilde{\boldsymbol{y}} \mid x_{i}, s\right) f(s) d s,
$$

where $f(s)$ is the probability density function (PDF) of $s$ and $\mathbb{S}$ denotes its signal space. It indicates the receiver is dependent upon the type of $s$. In the following, we derive the receivers for the deterministic-unknown $s$ and the Gaussian $s$, respectively.

1) deterministic-unknown ambient signal : When the prior information about $s$ is not available, the receiver obtains its estimate $\hat{s}$ from a measurement $\tilde{\boldsymbol{y}}$. In this case, the measurement $\tilde{\boldsymbol{y}}$ has a joint distribution of the estimation error $\epsilon=\hat{s}-s$ and the projected noise $\boldsymbol{G}_{0} \boldsymbol{n}$. Thus, Eq. (2) becomes

$$
f\left(\tilde{\boldsymbol{y}} \mid x_{i}\right)=\int_{\mathbb{S}_{\epsilon}} f\left(\tilde{\boldsymbol{y}} \mid x_{i}, \epsilon\right) f(\epsilon) d \epsilon
$$

where $\mathbb{S}_{\epsilon}$ is the support of $\epsilon$, and $f(\epsilon)$ is its PDF.

The receiver acquires the estimate of the ambient signal $\hat{s}$ from a temporal measurement $\tilde{\boldsymbol{y}}$ given $x_{i}$ is transmitted using the maximum likelihood estimator. The maximum likelihood estimate and the estimation error are

$$
\hat{s}=\frac{\boldsymbol{g}_{i}^{H} \boldsymbol{G}_{0} \tilde{\boldsymbol{y}}}{\left\|\boldsymbol{G}_{0} \boldsymbol{g}_{i}\right\|^{2}}, \quad \epsilon=\frac{\boldsymbol{g}_{i}^{H} \boldsymbol{G}_{0} \boldsymbol{n}}{\left\|\boldsymbol{G}_{0} \boldsymbol{g}_{i}\right\|^{2}} .
$$

The estimation error $\epsilon$ is a complex Gaussian random variable with mean $\mathrm{E}\{\epsilon\}=0$ and variance $\mathrm{E}\left\{\epsilon \epsilon^{*}\right\}=1 /\left\|\boldsymbol{G}_{0} \boldsymbol{g}_{i}\right\|^{2}$. Taking $f(\epsilon)$ into Eq. (3), $f\left(\tilde{\boldsymbol{y}} \mid x_{i}\right)$ can be calculated as

$$
\begin{aligned}
f\left(\tilde{\boldsymbol{y}} \mid x_{i}\right)=\frac{1}{2 \pi^{N_{r}}} \exp ( & -\frac{|\hat{s}|^{2}}{2}\left\|\boldsymbol{G}_{0} \boldsymbol{g}_{i}\right\|^{2}+\operatorname{Re}\left\{\tilde{\boldsymbol{y}}^{H} \boldsymbol{G}_{0} \boldsymbol{g}_{i} \hat{s}\right\} \\
& \left.+\frac{\tilde{\boldsymbol{y}}^{H} \boldsymbol{G}_{0} \boldsymbol{g}_{i} \boldsymbol{g}_{i}^{H} \boldsymbol{G}_{0} \tilde{\boldsymbol{y}}}{2\left\|\boldsymbol{G}_{0} \boldsymbol{g}_{i}\right\|^{2}}-\tilde{\boldsymbol{y}}^{H} \boldsymbol{G}_{0} \tilde{\boldsymbol{y}}\right) .
\end{aligned}
$$

Then, substituting the likelihood function into Eq. (1), the binary hypotheses testing can be simplified to

$$
\tilde{\boldsymbol{y}}^{H} \frac{\boldsymbol{G}_{0} \boldsymbol{g}_{1} \boldsymbol{g}_{1}^{H} \boldsymbol{G}_{0}}{\left\|\boldsymbol{G}_{0} \boldsymbol{g}_{1}\right\|^{2}} \tilde{\boldsymbol{y}} \underset{\mathcal{H}_{1}}{\stackrel{\mathcal{H}_{0}}{\lessgtr}} 2 \log \frac{p\left(x_{1}\right)}{p\left(x_{0}\right)}+\frac{1}{3}|s|^{2}\left\|\boldsymbol{G}_{0} \boldsymbol{g}_{1}\right\|^{2} .
$$

2) Gaussian ambient signal: The distribution of the ambient signal is assumed to be $s \sim \mathcal{C N}\left(0, \sigma_{s}^{2}\right)$. By integrating over the signal space of $s$ in Eq. (2), the likelihood function can be written as

$$
f\left(\tilde{\boldsymbol{y}} \mid x_{i}\right)=\frac{\exp \left(\tilde{\boldsymbol{y}}^{H} \boldsymbol{G}_{0} \tilde{\boldsymbol{y}}+\frac{\sigma_{s}^{2}\left|\tilde{\boldsymbol{y}} \boldsymbol{G}_{0} \boldsymbol{g}_{i}\right|^{2}}{1+\sigma_{s}^{2}\left\|\boldsymbol{G}_{0} \boldsymbol{g}_{i}\right\|^{2}}\right)}{\pi^{N_{r}}\left(1+\sigma_{s}^{2}\left\|\boldsymbol{G}_{0} \boldsymbol{g}_{i}\right\|^{2}\right)} .
$$

Substituting Eq. (6) into Eq. (1), and taking logarithm on both sides, the binary hypotheses testing for Gaussian $s$ is

$$
\begin{aligned}
\tilde{\boldsymbol{y}}^{H} \frac{\boldsymbol{G}_{0} \boldsymbol{g}_{1} \boldsymbol{g}_{1}^{H} \boldsymbol{G}_{0}}{\left\|\boldsymbol{G}_{0} \boldsymbol{g}_{1}\right\|^{2}} \tilde{\boldsymbol{y}} \stackrel{\mathcal{H}_{\mathcal{H}_{1}}}{\lessgtr} & \frac{1+\sigma_{s}^{2}\left\|\boldsymbol{G}_{0} \boldsymbol{g}_{1}\right\|^{2}}{\sigma_{s}^{2}\left\|\boldsymbol{G}_{0} \boldsymbol{g}_{1}\right\|^{2}} \log \frac{p\left(x_{0}\right)\left(1+\sigma_{s}^{2}\left\|\boldsymbol{G}_{0} \boldsymbol{g}_{1}\right\|^{2}\right)}{p\left(x_{1}\right)} .
\end{aligned}
$$

As can be seen from Eq. (5) and Eq.(7), the test statistics of the simplified receiver for the deterministic-unknown $s$ and the 
Gaussian $s$, denoted as $z_{d}$ and $z_{g}$ respectively, are the same and can be written as

$$
\begin{aligned}
z_{d} & =z_{g}=z \triangleq \tilde{\boldsymbol{y}}^{H} \frac{\boldsymbol{G}_{0} \boldsymbol{g}_{1} \boldsymbol{g}_{1}^{H} \boldsymbol{G}_{0}}{\left\|\boldsymbol{G}_{0} \boldsymbol{g}_{1}\right\|^{2}} \tilde{\boldsymbol{y}} \\
& =\boldsymbol{y}^{H} \frac{\boldsymbol{G}_{0} \boldsymbol{g}_{1} \boldsymbol{g}_{1}^{H} \boldsymbol{G}_{0}}{\left\|\boldsymbol{G}_{0} \boldsymbol{g}_{1}\right\|^{2}} \boldsymbol{y}=\left|\boldsymbol{b}^{H} \boldsymbol{y}\right|^{2},
\end{aligned}
$$

where the first equality in the second line arises from the fact that $\boldsymbol{G}_{0}$ is an idempotent matrix, and we denote $\boldsymbol{b}=$ $\boldsymbol{G}_{0} \boldsymbol{g}_{1} /\left\|\boldsymbol{G}_{0} \boldsymbol{g}_{1}\right\|$ as the normalized beamformer. Derived from MAP criterion, the beamformer $\boldsymbol{b}$ maximizes the difference between the signal under two hypotheses. The test statistic indicates that the receiver is an energy detector measuring the energy of a beamformed signal. Since, in this paper, we suppose that the prior probabilities are known, the energy detector makes a decision based on the thresholds that are derived from the Bayesian criterion. The thresholds for the deterministic-unknown $s$ and the Gaussian $s$ are expressed as

$$
\begin{gathered}
V_{d} \triangleq 2 \log \frac{p\left(x_{1}\right)}{p\left(x_{0}\right)}+\frac{1}{3}|s|^{2} \kappa^{2}, \\
V_{g} \triangleq \frac{1+\sigma_{s}^{2} \kappa^{2}}{\sigma_{s}^{2} \kappa^{2}} \log \frac{p\left(x_{0}\right)\left(1+\sigma_{s}^{2} \kappa^{2}\right)}{p\left(x_{1}\right)},
\end{gathered}
$$

where we denote $\kappa=\left\|\boldsymbol{G}_{0} \boldsymbol{g}_{1}\right\|$ as the magnitude of the component of $\boldsymbol{g}_{1}$ which is orthogonal to $\boldsymbol{g}_{0}$.

\section{B. Receiver performance}

The performance of the simplified receiver are quantified by the probability of false alarm $P_{f}$ and the probability of detection $P_{d}$, from which we can obtain the error probability $P_{e}$ of the receiver. These probabilities are given by

$$
\begin{aligned}
& P_{f}=\operatorname{Pr}\left\{z>V \mid x_{0}\right\}=1-F\left(z=V \mid x_{0}\right), \\
& P_{d}=\operatorname{Pr}\left\{z>V \mid x_{1}\right\}=1-F\left(z=V \mid x_{1}\right), \\
& P_{e}=p\left(x_{0}\right) P_{f}+p\left(x_{1}\right)\left(1-P_{d}\right),
\end{aligned}
$$

where $F(\cdot \mid x)$ is the cumulative density function (CDF) of the test statistic $z$ when $x$ is transmitted. Therefore, analyzing the performance of the simplified receiver requires the distributions of $z$ under two hypotheses.

1) deterministic-unknown ambient signal: The beamformed signal $\boldsymbol{b}^{H} \boldsymbol{y}$ when $x_{i}$ and $s$ are given is a Gaussian random variable with its mean and variance represented as

$$
\mu_{\boldsymbol{b}^{H} \boldsymbol{y} \mid x_{i}, s}=s \boldsymbol{b}^{H} \boldsymbol{g}_{i}, \quad \Sigma_{\boldsymbol{b}^{H} \boldsymbol{y} \mid x_{i}, s}=1 .
$$

since the beamformer is a constant. When $\mathcal{H}_{0}$ is true, $\mu_{\boldsymbol{b}^{H} \boldsymbol{y} \mid x_{i}, s}=0$. Thus, $z_{d}$ has a chi-square distribution with 2 degrees of freedom and common variance $1 / 2$ [12, ch. 2]. Then, the PDF of $z_{d}$ under $\mathcal{H}_{0}$ is given by

$$
f_{\chi}\left(z_{d} \mid x=x_{0}\right)=\exp \left(-z_{d}\right) .
$$

If $\mathcal{H}_{1}$ is true, $z_{d}$ is a non-central chi-square distributed variable with 2 degrees of freedom, common variance $1 / 2$ and noncentrality parameter $|s|^{2}\left|\boldsymbol{b}^{H} \boldsymbol{g}_{1}\right|^{2}=|s|^{2} \kappa^{2}$ [12, ch. 2]. Hence, the PDF of $z_{d}$ under $\mathcal{H}_{1}$ is given by

$$
f_{\chi^{\prime}}\left(z_{d} \mid x=x_{1}\right)=\exp \left(-\left(z_{d}+|s|^{2} \kappa^{2}\right)\right) I_{0}\left(2 \sqrt{|s|^{2} \kappa^{2} z_{d}}\right),
$$

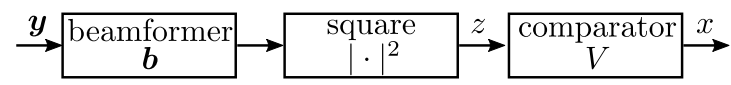

Fig. 2. Diagram of the simplified optimum receiver.

where $I_{v}(\cdot)$ is the vth order modified Bessel function of the first kind [13, sec. 9.6].

With density functions of the test statistic under two hypotheses, $P_{f}$ and $P_{d}$ for a given threshold $V_{d}$ are

$$
\begin{aligned}
& P_{f}=\operatorname{Pr}\left\{z>V_{d} \mid \mathcal{H}_{0}\right\}=\exp \left(-V_{d}\right), \\
& P_{d}=\operatorname{Pr}\left\{z>V_{d} \mid \mathcal{H}_{1}\right\}=Q_{1}\left(\sqrt{2|s|^{2} \kappa^{2}}, \sqrt{2 V_{d}}\right),
\end{aligned}
$$

where $Q_{1}(\cdot, \cdot)$ is the generalized Marcum $Q$-function.

2) Gaussian ambient signal: The beamformed signal $\boldsymbol{b}^{H} \boldsymbol{y}$ when $x=x_{i}$ is transmitted follows the Gaussian distribution of which mean and variance are

$$
\mu_{\boldsymbol{b}^{H} \boldsymbol{y} \mid x_{i}}=0, \quad \Sigma_{\boldsymbol{b}^{H} \boldsymbol{y} \mid x_{i}}=\boldsymbol{b}^{H} \boldsymbol{R}_{i}^{(g)} \boldsymbol{b},
$$

where $\boldsymbol{R}_{i}^{(g)}=\sigma_{s}^{2} \boldsymbol{g}_{i} \boldsymbol{g}_{i}^{H}+\boldsymbol{I}$ is the covariance matrix of $\boldsymbol{y}$ given $x=x_{i}$. Since the mean is always 0 , the random variable $z$ under two hypotheses follows the chi-square distribution both with degrees of freedom 2 , but with different common variance. Under hypothesis $\mathcal{H}_{0}, \Sigma_{\boldsymbol{b}^{H}} \boldsymbol{y} \mid x_{0}=1$, the PDF of $z_{g}$ is given by Eq. (10). When $\mathcal{H}_{1}$ is true, the common variance is $\left(1+\sigma_{s}^{2} \kappa^{2}\right) / 2$. Thus, the PDF is

$$
f_{\chi}\left(z_{g} \mid x=x_{1}\right)=\frac{1}{1+\sigma_{s}^{2} \kappa^{2}} \exp \left(\frac{z}{1+\sigma_{s}^{2} \kappa^{2}}\right) .
$$

For the density probability functions of $z_{g}$ under two hypotheses, $P_{f}$ and $P_{d}$ are given by

$$
\begin{aligned}
& P_{f}=\operatorname{Pr}\left\{z_{g}>V_{g} \mid \mathcal{H}_{0}\right\}=\exp \left(-V_{g}\right), \\
& P_{d}=\operatorname{Pr}\left\{z_{g}>V_{g} \mid \mathcal{H}_{1}\right\}=\exp \left(-\frac{V_{g}}{1+\sigma_{s}^{2} \kappa^{2}}\right) .
\end{aligned}
$$

\section{IMPLEMENTATION}

In this section, practical issues about implementation are provided, followed by an estimation method for required parameters. Then, key parameters that affect the receiver performance and a comparison with two receivers are discussed.

1) Practical implementation: The simplified receiver has a simple structure whose test statistic is independent of the ambient signal type, but the threshold varies. The implementation of its structure is shown in Fig. 2. The received signal $y$ goes through the beamformer $\boldsymbol{b}$, followed by feeding into the squaring device. Then, the obtained signal energy is compared with the threshold $V$ for inferring the BD signal.

The test statistic and the decision threshold required for implementing the receiver are the functions of two signal directions $\boldsymbol{g}_{i}, i \in\{0,1\}$ and the transmit power $P_{s}$. These parameters can be estimated from the auto-correlation matrix of the received signal. For the deterministic-unknown ambient signal and the Gaussian ambient signal, the auto-correlation matrices of received signal $\boldsymbol{y}$ when $x_{i}$ is transmitted are

$$
\boldsymbol{R}_{\boldsymbol{y} \mid x_{i}}^{(d)}=|s|^{2} \boldsymbol{g}_{i} \boldsymbol{g}_{i}^{H}+\boldsymbol{I}, \quad \boldsymbol{R}_{\boldsymbol{y} \mid x_{i}}^{(g)}=\sigma_{s}^{2} \boldsymbol{g}_{i} \boldsymbol{g}_{i}^{H}+\boldsymbol{I} .
$$


It can be observed that, $\lambda_{i}^{(d)}=1+|s|^{2}\left\|\boldsymbol{g}_{i}\right\|^{2}$, and $\lambda_{i}^{(g)}=$ $1+\sigma_{s}^{2}\left\|\boldsymbol{g}_{i}\right\|^{2}$ are the largest eigenvalues for these two autocorrelation matrices, respectively, and $\tilde{\boldsymbol{g}}_{i} \triangleq \boldsymbol{g}_{i} /\left\|\boldsymbol{g}_{i}\right\|$ is the associated eigenvector. Thus, the problem requires the estimation of auto-correlation matrices, their largest eigenvalues and the associated eigenvectors.

Let us denote the preamble sequences transmitted from the $\mathrm{BD}$ as $x[1]=\cdots=x[L]=x_{0}$ and $x[L+1]=\cdots=$ $x[2 L]=x_{1}$, and the corresponding received signal matrix as $\boldsymbol{Y}_{i}=[\boldsymbol{y}[1+i L] \cdots \boldsymbol{y}[L+i L]]$. Then, the auto-correlation matrix can be estimated as

$$
\hat{\boldsymbol{R}}_{\boldsymbol{y} \mid x_{i}}=\frac{1}{L} \sum_{l=1}^{L} \boldsymbol{y}[l+i L] \boldsymbol{y}[l+i L]^{H}=\frac{1}{L} \boldsymbol{Y}_{i} \boldsymbol{Y}_{i}^{H} .
$$

We assume the preamble length $L$ is selected so that $\hat{\boldsymbol{R}}_{\boldsymbol{y} \mid x_{i}} \approx$ $\boldsymbol{R}_{\boldsymbol{y} \mid x_{i}}$. Then, one can estimate the eigenvector associated with the largest eigenvalue using the power iteration method [14, Sec. 8.2.1], which is described by

$$
\boldsymbol{u}_{k+1}=\frac{\boldsymbol{Y}_{i} \boldsymbol{Y}_{i}^{H} \boldsymbol{u}_{k}}{\left\|\boldsymbol{Y}_{i} \boldsymbol{Y}_{i}^{H} \boldsymbol{u}_{k}\right\|},
$$

for the iteration number $k=0,1, \cdots$ and $\boldsymbol{u}_{k}$ is the $k$ th eigenvector estimate. The iteration stops when the estimate satisfies the convergence criterion. With the eigenvector estimate, the largest eigenvalue is calculated by $\hat{\lambda}=\frac{1}{L} \boldsymbol{u}_{k}^{H} \boldsymbol{Y}_{i} \boldsymbol{Y}_{i}^{H} \boldsymbol{u}_{k}$.

The power iteration method converges under two assumptions. One is that the starting vector $\boldsymbol{u}_{0}$ has a non-zero component in the direction of the eigenvector to be estimated. In practical AmBC systems, $\boldsymbol{u}_{0}$ can be set to an estimate of $\boldsymbol{\alpha}$, which can be obtained when the BD does not operate. The other is the largest eigenvalue is unique and is strictly larger than the other eigenvalues, which is fulfilled given high enough $|s|^{2}\left\|\boldsymbol{g}_{i}\right\|^{2}$ and $\sigma_{s}^{2}\left\|\boldsymbol{g}_{i}\right\|^{2}$.

Therefore, utilizing the estimated largest eigenvalues and the associated eigenvectors, the test statistic in Eq. (8) and the decision thresholds in Eq. (9) can be calculated by substituting the following parameters

$$
\begin{gathered}
\boldsymbol{b}=\left(\boldsymbol{I}-\tilde{\boldsymbol{g}}_{0} \tilde{\boldsymbol{g}}_{0}^{H}\right) \tilde{\boldsymbol{g}}_{1} /\left\|\left(\boldsymbol{I}-\tilde{\boldsymbol{g}}_{0} \tilde{\boldsymbol{g}}_{0}^{H}\right) \tilde{\boldsymbol{g}}_{1}\right\| \\
|s|^{2} \kappa^{2}=\left(\lambda_{1}^{(d)}-1\right)\left(1-\left|\tilde{\boldsymbol{g}}_{0}^{H} \tilde{\boldsymbol{g}}_{1}\right|^{2}\right) \\
\sigma_{s}^{2} \kappa^{2}=\left(\lambda_{1}^{(g)}-1\right)\left(1-\left|\tilde{\boldsymbol{g}}_{0}^{H} \tilde{\boldsymbol{g}}_{1}\right|^{2}\right)
\end{gathered}
$$

2) Discussion: The performance of the simplified receiver is defined by the distributions of the test statistic under two hypotheses. The distributions deviate from each other as $|s|^{2} \kappa^{2}$ increases for deterministic-unknown $s$, and as $\sigma_{s}^{2} \kappa^{2}$ increases for Gaussian $s$, respectively. These two parameters are quantified by the transmit power $P_{s}$ and the parameter $\kappa^{2}$ which measures the component of $\boldsymbol{g}_{1}$ that is orthogonal to $\boldsymbol{g}_{0}$. Theoretically, $\kappa^{2}$ reaches the maximum when $\boldsymbol{g}_{0}$ is perpendicular to $\boldsymbol{g}_{1}$. However, in AmBC systems, these two signal directions cannot be perpendicular since the backscatter path is much weak compared with the direct path. Moreover, $\kappa^{2}$ is minimum when $\boldsymbol{g}_{0}$ and $\boldsymbol{g}_{1}$ are in the same direction, which happens when the BD is at the line between the Tx and the Rx. Therefore, the simplified receiver achieves an acceptable performance when the $\mathrm{BD}$ is at a suitable location.

It is worth noting that a beamforming-assisted energy detection has been proposed for the Gaussian ambient signal and OOK-modulated BD signal in the recent work [3]. Compared with [3], we give a closed-form expression of the optimal beamformer $\boldsymbol{b}$ which maximizes the difference of signal energies under two hypotheses, and we further analyze the performance of the proposed receiver. Compared with the optimum receiver in [4], the number of RF fronts ends needed to implement the receiver can be halved. Moreover, the beamformers can be implemented in the analog domain to mitigate the dynamic range problem.

\section{NUMERICAL RESULTS}

In this section, we present and discuss several Monte Carlo simulation results of the proposed simplified receiver.

The distances are wavelength-scaled so that the results are carrier-frequency independent. The simulation scenario is illustrated in Fig. 1. A linear array with $\lambda / 2$ of antenna separation is considered at the Rx. The coordinates of the $\mathrm{Rx}$ reference antenna and the Tx antenna are $[-40 \lambda, 0]$ and $[40 \lambda, 0]$. The coordinate the BD is $[(-40+3 / \sqrt{2}) \lambda,-3 \lambda / \sqrt{2}]$. The power loss induced by the BD modulator is $\rho=0.6$. Hence, the difference between the SNR of ambient signal and the effective SNR of backscatter signal is $\gamma-\gamma_{e} \approx 33.5 \mathrm{~dB}$.

The BD symbol is drawn from the discrete uniform distribution, i.e., $p\left(x_{0}\right)=p\left(x_{1}\right)=0.5$. BPSK modulation is considered at the BD. The simplified receiver for the deterministicunknown ambient signal is referred to as deterministic receiver where $s$ is generated from QPSK-modulated signal, and the simplified receiver for the Gaussian ambient signal is referred to as Gaussian receiver where $s$ is generated from a zeromean complex Gaussian distribution. The transmit power is $P_{s}=\gamma N_{r} /\|\boldsymbol{\alpha}\|^{2}$.

In Fig. 3, the performance of the simplified receiver is evaluated in terms of BER. The impact of the number of receiver antennas $N_{r}$ on BER performance of two receivers is shown in Fig. 3a. The improvement of BER is diminishing as $N_{r}$ becomes larger. Especially for the Gaussian receiver, the BER does not improve when $N_{r} \geq 16$, which is aligned with the performance of receive diversity [11, Sec. 3.3.1]. Thus, we select $N_{r}=16$ considering the Gaussian receiver.

In Fig. 3b, BER performance of Gaussian receiver and deterministic receiver as a function of the preamble length $L$ is compared when the receivers are implemented with known beamformer and threshold and estimated from power iteration (p.i.). For the Gaussian receiver, the performance difference between the two implementations is negligible for $L \geq N_{r}$. For the deterministic receiver, the implementation with p.i. estimation suffers from a small performance loss, which decreases as $L$ increases. Therefore, we select the power iteration method and we fix $L=32$ to obtain an accurate enough estimated beamformer and threshold.

In Fig. 3c, the BER performance of the Gaussian receiver and the deterministic receiver are compared with the optimum 


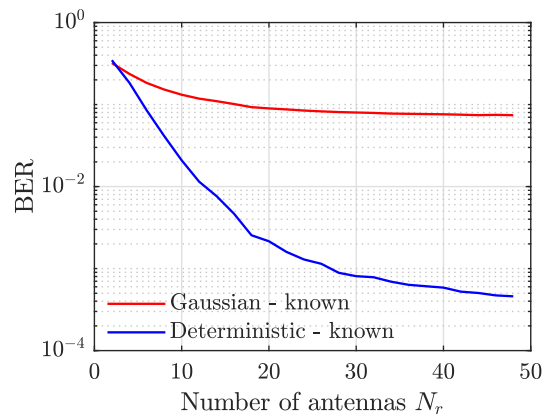

(a)

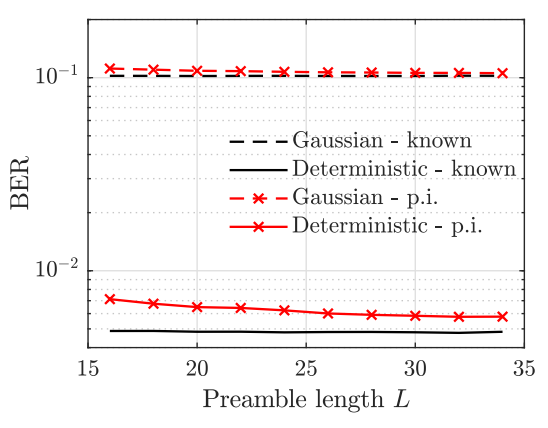

(b)

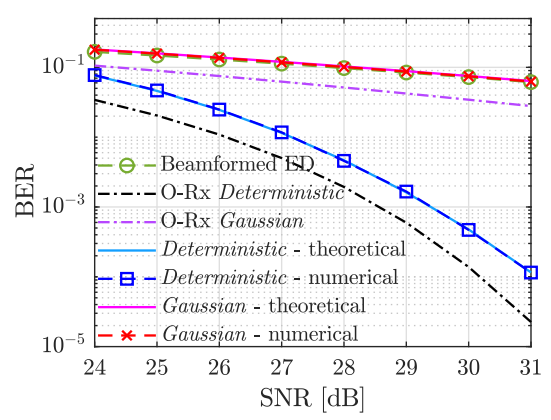

(c)

Fig. 3. Performance evaluation of the simplified receiver in terms of bit error rate (BER). In (a), variation in BER as a function of the number of receiver antennas $N_{r}$ for the Gaussian receiver and the deterministic receiver for the SNR of the ambient signal $\gamma=28 \mathrm{~dB}$ when the beamformer and the threshold are known. In (b), variation in BER with the preamble length $L$ for the Gaussian receiver and the deterministic receiver for $\gamma=28 \mathrm{~dB}$ and $N_{r}=16$ when the beamformer and the threshold are known and are estimated from power iteration (p.i.). In (c), comparison of BER as a function of SNR $\gamma$ for $N_{r}=16$ between the numerical BER of the beamformed energy detection (ED) in [3], the theoretical BER of the optimum receiver (O-Rx) of two types of ambient signal in [4], and the theoretical BER and numerical BER of simplified receiver for two types of ambient signal.

receivers in [4] and the beamformed energy detection (ED) in [3]. As can be seen, for two proposed receivers, the derived theoretical error probabilities are very close to the corresponding numerical BERs which are obtained with known beamformer and threshold. This verifies that the performance analysis for the two receivers is valid. As discussed earlier, the beamformed ED proposed in [3] for the Gaussian $s$ has the same performance as the Gaussian receiver. Compared with the optimum receivers in [4], the Gaussian receiver requires around 3.6-dB more of SNR to achieve the same performance as the optimum receiver, while the deterministic receiver only requires around $1-\mathrm{dB}$ more of SNR.

\section{CONCLUSION}

In this paper, we propose a simplified multi-antenna AmBC receiver for any binary-modulated backscatter signal. The test statistics of the receiver for the deterministic-unknown ambient signal and the Gaussian ambient signal, which are derived from the MAP criterion, are the same. It contains one beamformer that maximizes the energy difference of the signal under two hypotheses. The simplified receiver opens up possibilities for addressing practical implementation issues. In particular, it saves half of the components at the cost of SNR penalty as low as 1-dB to achieve the same performance as the optimum receiver for the deterministic-unknown ambient signal and 3.6-dB for the Gaussian ambient signal. It can also mitigate the dynamic range issue by being implemented in the analog domain. Therefore, the proposed simplified receiver is a costeffective solution for implementing $\mathrm{AmBC}$ receiver.

\section{ACKNOWLEDGMENT}

This work is partially supported by the Academy of Finland under Project No. 319003, and the China Scholarship Council.

\section{REFERENCES}

[1] V. Liu, A. Parks, V. Talla, S. Gollakota, D. Wetherall, and J. R. Smith, "Ambient backscatter: Wireless communication out of thin air," in Proceedings of the ACM SIGCOMM, Hong Kong, China, 2013, pp. $39-50$.
[2] A. N. Parks, A. Liu, S. Gollakota, and J. R. Smith, "Turbocharging ambient backscatter communication," in Proceedings of the ACM SIGCOMM, Chicago, Illinois, USA, 2014, pp. 619-630.

[3] H. Guo, Q. Zhang, S. Xiao, and Y. Liang, "Exploiting multiple antennas for cognitive ambient backscatter communication," IEEE Internet Things Journal, vol. 6, no. 1, pp. 765-775, Feb 2019.

[4] H. Yiğitler, X. Wang, and R. Jäntti, "Optimum multi-antenna ambient backscatter receiver for binary-modulated tag signals," 2020.

[5] M. A. ElMossallamy, M. Pan, R. Jäntti, K. G. Seddik, G. Y. Li, and Z. Han, "Noncoherent backscatter communications over ambient ofdm signals," IEEE Transactions on Communications, vol. 67, no. 5, pp. 3597-3611, 2019.

[6] G. Yang, Q. Zhang, and Y. Liang, "Cooperative ambient backscatter communications for green Internet-of-Things," IEEE Internet Things Journal, vol. 5, no. 2, pp. 1116-1130, Apr. 2018.

[7] R. Duan, E. Menta, H. Yiğitler, R. Jäntti, and Z. Han, "Hybrid beamformer design for high dynamic range ambient backscatter receivers," in IEEE International Conference on Communications Workshops, 2019, pp. 1-6.

[8] G. Yang, Y.-C. Liang, R. Zhang, and Y. Pei, "Modulation in the air: backscatter communication over ambient OFDM carrier," IEEE Transactions on Communications, vol. 66, no. 3, pp. 1219-1233, Mar. 2018.

[9] Q. Tao, C. Zhong, X. Chen, H. Lin, and Z. Zhang, "Optimal detection for ambient backscatter communication systems with multiantenna reader under complex gaussian illuminator," IEEE Internet of Things Journal, vol. 7, no. 12, pp. 11371-11383, 2020.

[10] X. Wang, H. Yiğitler, R. Duan, E. Y. Menta, and R. Jäntti, "Coherent multi-antenna receiver for bpsk-modulated ambient backscatter tags," IEEE Internet of Things Journal, 2021, in press.

[11] D. Tse and P. Viswanath, Fundamentals of wireless communication. Cambridge university press, 2005.

[12] J. Proakis and M. Salehi, Digital Communications, 5th ed. McGraw-Hill, 2008.

[13] M. Abramowitz and I. A. Stegun, Handbook of mathematical functions: with formulas, graphs, and mathematical tables. Dover Publications, 1970.

[14] G. H. Golub and C. F. Van Loan, Matrix computations, 4th ed. Johns Hopkins University Press Baltimore, 2013. 